Review

\title{
Hallux valgus - a case for a physiotherapist or only for a surgeon? Literature review
}

\author{
Kamila MortKa, MSc1 ${ }^{*}$, PrZemysŁaW Lisiński, MD, PhD ${ }^{1)}$ \\ 1) Clinic of Rehabilitation, Department of Rheumatology and Rehabilitation, Poznan University of \\ Medical Sciences: ul. 28 Czerwca 1956r. nr 135/147, 61-545 Poznań, Poland
}

\begin{abstract}
Purpose] Hallux valgus is one of the most common deformations of the human foot, and it causes great difficulties for the patients. The aim of this paper was to review available medical literature in search of evidence which would justify implementation of physiotherapy, based on its effectiveness for patients with hallux valgus. [Subjects and Methods] The following databases were searched for applicable papers: PubMed, Google Scholar, Clinical Key and UpToDate. Full-text articles from the last 15 years were subjected to a review, which ultimately selected seven papers about hallux valgus therapy published over the past 12 years. These studies were grouped according to their design and level of evidence and classified depending on whether they concerned physiotherapy as postoperative therapy or as the only treatment. [Results] The studies included in the present analysis used the following interventions for patients with hallux valgus: exercise, manual therapy, gait training, taping and orthosis. All the studies showed beneficial effects and the most frequently observed results were reductions in pain and improvements in function. [Conclusion] The evidence found in the reviewed materials clearly indicates that patients with hallux valgus should not only be subject to surgical procedures but also undergo physiotherapeutic treatment. Key words: Hallux valgus, Rehabilitation, Physiotherapy
\end{abstract}

(This article was submitted Apr. 24, 2015, and was accepted Jun. 25, 2015)

\section{INTRODUCTION}

Hallux valgus is one of the most common deformations of the human foot. It causes pain, walking difficulties and can be problematic for choosing the proper footwear. According to the definition it is described as a lateral deviation of the great toe at the metatarsophalangeal joint. Hallux valgus is diagnosed when the hallux valgus angle is greater than 15 degrees ${ }^{1)}$.

A systematic review of 78 studies involving 496,957 subjects showed that the prevalence of hallux valgus is approximately 23 percent among adults aged 18 to 65 years, 36 percent among adults over 65 years, and even 30 percent among adult females ${ }^{2}$.

It is universally accepted that hallux valgus arises in steps, which do not necessarily occur in series but may occur in parallel. The precise etiology of hallux valgus deformity is unknown. Much research has confirmed the multifactorial origins of hallux valgus, which include several predisposing extrinsic factors such as high-heeled narrow shoes and excessive weight-bearing. The intrinsic factors are: genetics, ligamentous laxity, metatarsus primus varus, pes planus, functional hallux limitus, sexual dimorphism, age, abnormal

*Corresponding author. Kamila Mortka (E- mail: mortkakamila@gmail.com)

C2015 The Society of Physical Therapy Science. Published by IPEC Inc. This is an open-access article distributed under the terms of the Creative Commons Attribution Non-Commercial No Derivatives (by-ncnd) License $<$ http://creativecommons.org/licenses/by-nc-nd/3.0/>. metatarsal morphology, first-ray hypermobility and tight Achilles tendon ${ }^{3)}$.

Many treatments for hallux valgus deformity have been considered in numerous studies. Medical literature focuses primarily on the surgical treatment of this deformity. Surgical intervention is the most common approach with over 150 different procedures ${ }^{2}$. The effectiveness of conservative treatment of hallux valgus is often ignored. Unfortunately the operative methods can have unpredictable and equivocal outcomes. The key to surgical success is proper timing and the type of surgical procedure. Poor results of surgery have been noted in cases of generalized joint laxity and hypermobility ${ }^{4}$. Additionally, surgery carries the risk of post-operative complications such as scars, infection, recurrence of pain or deformity, worsening of pain or deformity, over-correction, stiffness, weakness, neurovascular damage, deep vein thrombosis, pulmonary embolism, bone or fixation device failure, prolonged swelling and healing, malunion or nonunion of osteotomy, difficulty with walking or loss of function, etc ${ }^{5-7}$. In hallux valgus surgery the complication rate ranges between $10 \%$ and $50 \%{ }^{8}$ ).

The purpose of the study was to justify implementation of physiotherapy in hallux valgus deformation treatment and to judge its effects based on the evidence presented in available Polish and foreign medical literature. This work should answer the following questions:

- Is a patient with hallux valgus a case for physiotherapist? Is the implementation of physiotherapy in treatment of this deformity beneficial and effective?

- When may physiotherapy be implemented? Should it be considered (alone or with other conservative treatments) 
as the basic treatment, or is it essential only in the postoperative period?

- What method of physiotherapy has the best therapy outcomes?

\section{SUBJECTS AND METHODS}

The following databases were searched for appropriate papers: PubMed, PEDro, Clinical Key and UpToDate. The search terms were as follows: hallux valgus, hallux abducto valgus, hallux abducto valgus in connection with: physiotherapy, rehabilitation, physical therapy, conservative treatment, post-operative treatment, manual therapy, exercise, taping. Study subjects with a diagnosis of hallux valgus were the most important inclusion criterion in this review. Other criteria were studies written in Polish, English, or an English abstract. Full-text articles published in the last 15 years were taken into consideration. Study subjects with rheumatological or neurological factors were the exclusion criteria of this work.

The studies retrieved were grouped according to their design and the level of evidence into randomized controlled trials (RCT), controlled trials which were not randomized and experimental trials or case studies.

The studies were additionally divided into two classifications: post-operative therapy, and physiotherapy as the only treatment for patients with hallux valgus. Further analyses included: the methods of physiotherapy, the number of participants, presence of a control group, the duration of the therapy, outcome measures, and the results of treatment.

Only seven publications about hallux valgus therapy published in the last 12 years fulfilled the inclusion criteria and were analysed.

\section{RESULTS}

General analysis is presented in Table 1, and the details are described below.

The effects of rehabilitation after hallux valgus surgery were studied by Schuh et $\mathrm{al}^{8}$. They analysed the changes in plantar pressure distribution during the stance phase of gait in patients who received physical therapy and gait training in the postoperative period. This was a prospective descriptive study. The material included thirty participants who underwent Austin and scarf osteotomy for correction of mild to moderate hallux valgus deformity.

The therapy was based on multimodal rehabilitation program, which initially included: an Aircast cryo-cuff (applied for 8 hours starting immediately after surgery on the day of the operation and on the first postoperative day), the Rathgeber postoperative shoe (for 4 weeks) and a special sock, Gilofamed, that reduces swelling and the need for dressing changes. Then, 4 weeks after surgery, the first session of physical therapy was implemented and it was continued with a frequency of once per week.

To reduce swelling of the leg, elevation, lymphatic drainage, activation of the muscle pump, and cryotherapy (cool packs) were used. The aim of the gait training was to achieve physiological gait patterns. During the stance phase, participants trained to strike the heel in its physiological position at the lateral aspect of the heel. In midstance and the terminal stance, weight bearing on the first metatarsal was stressed. This was done with training of active push-off by the great toe flexors, the flexor digitorum longus and brevis muscles, and the lumbrical muscles.

The next stage of rehabilitation was selective strengthening of the peroneus longus muscle, whose function is to pronate the midfoot and it is responsible for ground contact of the first ray, the most heavily loaded structure of the foot during gait. When this muscle is weak, compensation in the form of valgus positioning of the knees can appear. Fascial release techniques for the peroneal muscles were also performed. To decrease the tone of the tibialis anterior muscle, velocity dependent resistance to stretch was used.

Manual therapy aimed to improve the range of motion of all metatarsophalangeal joints (MTP), and it included caudal sliding of the proximal phalanx (to improve flexion), and dorsal sliding of the proximal phalanx (to improve extension). Moreover, to activate the mechanoreceptors that restrain the afferent pain sensors the oscillating traction was implemented. Mobilization of the first MTP, Lisfranc, transverse tarsal, subtalar and ankle joints was also performed, and concentric strengthening exercises of the great toe flexors and extensors were included in the treatment protocol.

Schuh et al. reported an increase in plantar pressure parameters in the region of the great toe and the first ray after hallux valgus surgery in patients who received physical therapy as well as gait training in the post-operative period. They cited articles ${ }^{9-13)}$ that studied changes in plantar pressure distribution after hallux valgus surgery and found decreased loading parameters in the hallux and the first metatarsal head region after surgery. These studies did not include physical therapy or gait training. Therefore, Schuh et al. ${ }^{8)}$ suggested that postoperative physical therapy and gait training may lead to improved function and weight bearing of the first ray after hallux valgus surgery. However a major limitation of their study was the absence of a control group.

The effectiveness of manual therapy for patients with hallux valgus was analysed by du Plessis et $\mathrm{al}^{6}{ }^{6}$. Their study was a parallel-group randomised trial which aimed to test an innovative protocol of manual and manipulative therapy (MMT) and compare it with a treatment of a night splint(s) for symptomatic mild to moderate hallux valgus deformity. Fifteen participants in the control group used a night splint(s) and the second experimental group $(n=15)$ received a structured protocol of MMT (4 treatments over a 2-week period). The MMT protocol was based on the Brantingham protocol and included graded mobilisation of the affected hallux/1st MTPJ, judicious manipulation of the affected hallux/1st MTPJ, post-treatment cold therapy and additional mobilisation/manipulation of foot and ankle joints. The outcomes were measured using a visual analogue scale, foot function index and hallux dorsiflexion (goniometry). The results show that there were no statistically or clinically significant differences between the two groups, suggesting that manipulative therapy (experimental group) was equivalent to standard care of a night splint(s) (control group) for symptomatic mild to moderate hallux valgus in the short term.

Conservative treatment through manual therapy and specific foot exercises was presented by Jedynak ${ }^{14}$ in a 
Table 1. General analysis of the research material. A comparison of the studies, interventions, number of subjects, designs, duration or frequency of the therapies, outcome measures and results

\begin{tabular}{|c|c|c|c|c|c|c|}
\hline Study & Intervention & $\begin{array}{l}\text { Number } \\
\text { of study } \\
\text { material }\end{array}$ & Design & $\begin{array}{c}\text { Duration/ } \\
\text { frequency of the } \\
\text { therapy }\end{array}$ & Outcome measures & Results \\
\hline $\begin{array}{l}\text { Schuh } \\
2009\end{array}$ & $\begin{array}{l}\text { Multimodal rehabilitation } \\
\text { program (MRP): Cryo- } \\
\text { therapy, Exercise, Manual } \\
\text { therapy, Gait training }\end{array}$ & 30 & ET & $\begin{array}{l}4 \text { weeks after sur- } \\
\text { gery: MRP once } \\
\text { per week for } 4 \text { to } 6 \\
\text { weeks }\end{array}$ & $\begin{array}{l}\text { Plantar pressure analysis preopera- } \\
\text { tively and } 4 \text { weeks, } 8 \text { weeks, and } 6 \\
\text { months postoperatively }+ \text { ROM of } \\
\text { MTP, AOFAS preoperatively and at } \\
6 \text { months after surgery }\end{array}$ & $\begin{array}{c}\uparrow \mathrm{AOFAS}, \\
\uparrow \mathrm{ROM}, \\
\uparrow \text { plantar pressure } \\
\text { analysis }\end{array}$ \\
\hline $\begin{array}{l}\mathrm{du} \\
\text { Plessis } \\
2009\end{array}$ & $\begin{array}{c}\text { Manual therapy (MT) vs. } \\
\text { night splints (NS) }\end{array}$ & $\begin{array}{l}15 \text { in each } \\
\text { group }\end{array}$ & RCT & $\begin{array}{l}4 \text { times in } 2 \text { weeks } \\
\text { after } 1 \text { month } \\
\text { follow-up }\end{array}$ & FFI, VAS, HDF & $\begin{array}{c}\downarrow \text { pain } \uparrow \text { function } \\
\text { MT }=\mathrm{NS}, \\
\text { after } 1 \text { mo MT>NS }\end{array}$ \\
\hline $\begin{array}{l}\text { Jedy- } \\
\text { nak } \\
2009\end{array}$ & $\begin{array}{c}\text { Manual therapy and } \\
\text { exercise }\end{array}$ & 1 & CS & $\begin{array}{l}\text { MT three times } \\
\text { per week for three } \\
\text { months, exercise } \\
\text { for } 6 \text { months }\end{array}$ & FSHQ, IM angle, $\mathrm{HV}$ angle & $\downarrow$ pain $\uparrow$ function \\
\hline $\begin{array}{l}\text { Bayar } \\
2011\end{array}$ & Exercise and Taping & 10 & $\mathrm{RCT}$ & 8 weeks & HV angle, VAS, WAS & $\begin{array}{c}\text { taping and exercise }> \\
\text { exercise alone.: } \\
\downarrow \text { HV angle, } \downarrow \text { VAS, } \\
\downarrow \text { walking pain }\end{array}$ \\
\hline $\begin{array}{l}\text { Jeon } \\
2004\end{array}$ & Taping & $15 ; 25$ feet & ET & $\begin{array}{c}15 \text { treatments in } 4 \\
\text { weeks }\end{array}$ & Pain and $\mathrm{HV}$ angle & $\downarrow$ pain $\& \downarrow \mathrm{HV}$ angle \\
\hline $\begin{array}{l}\text { Bek } \\
\text { Kurklu } \\
2002\end{array}$ & $\begin{array}{l}\text { Manual therapy (MT), } \\
\text { toe separator (TS), night } \\
\text { splints (NS) }\end{array}$ & 15 & RCT & 3 months & VAS, HVI & $\begin{array}{c}\text { MT: } \downarrow \text { pain and } \\
\uparrow \text { correction of defor- } \\
\text { mity; } \\
\text { NS: } \downarrow \text { pain }\end{array}$ \\
\hline $\begin{array}{l}\text { Kim } \\
2013\end{array}$ & $\begin{array}{l}\text { Exercise: Short Foot (SF) } \\
\text { vs. Toe-Spread-Out (TSO) }\end{array}$ & $\begin{array}{l}18 \text { subjects } \\
\text { with mild } \\
\text { HV }\end{array}$ & $\mathrm{RCT}$ & 2 weeks & $\begin{array}{l}\text { muscle activity of AbdH and AddH } \\
\text { and the angle of MTP }\end{array}$ & $\begin{array}{l}\text { AbdH: TSO }>\text { SF; } \\
\text { ratio of } \mathrm{AbdH} \text { to } \\
\text { AddH: TSO }>\mathrm{SF} ; \\
\text { MTP angle: } \mathrm{TSO}>\mathrm{SF}\end{array}$ \\
\hline
\end{tabular}

Intervention (MRP: Multimodal Rehabilitation Program, MT: Manual Therapy; NS: night splints; TS: toe separator; SF: short foot; TSO: toe spread-out) Design (RCT: randomized controlled trial; ET: experimental trial; CS: case study) Outcome measures (ROM: range of motion, AOFAS: the metatarsophalangeal interphalangeal score of the American Orthopaedic Foot and Ankle Society, FSHQ: foot health status questionnaire for pain and function; FFI: foot function index, VAS: visual analogue scale, HDF: hallux dorsiflexion, WAS: walking ability scale, HVI: Hallux Valgus Index)

case study. A patient was treated using foot mobilisation techniques (FMT) which included: anterior calcaneal thrust, posterior talar mobilisation, lateral navicular manipulation, cuneiform-1st metatarsal mobilisation, 1st metatarsophalangeal joint (MTPJ) mobilisation and medial sesamoid mobilisation. These manual interventions were performed at a frequency of three times per week for three months. Over a six-month period, specific foot exercises: isometric contraction of the intrinsic muscles of the foot to maximum tolerance held for 20 seconds (three repetitions twice daily), isometric contraction of the extrinsic muscles of the foot to maximum tolerance held for 20 seconds (three repetitions twice daily), and dynamic adduction and release of the abductor hallucis with five-second holds (five repetitions twice daily). The exercises were based on the physiotherapeutic principles of strengthening and re-training these muscles, which are responsible for stabilisation of the tarsal and metatarsal bones of the feet. The following outcome measures were used: FHSQ (foot health status questionnaire for pain and function), IM angle (intermetatarsal angle), and HA angle (hallux abductus angle). The FHSQ results showed an improvement in both foot pain and foot function measurements at three and six months. Further radiographic measurements indicated there was a slight reduction in the IM angle of both feet and the HA angle of one of them.

The effects of a combined treatment program consisting of exercise and taping for patients with hallux valgus were assessed in a preliminary study of Bayar et $\mathrm{al}^{15)}$. Twenty female patients with a diagnosis of hallux valgus were included in the research. The subjects were randomly divided into 2 groups (each containing 10 subjects): a group which was treated with taping and foot exercises and a control group which did only the foot exercises. The researchers assessed the following parameters: hallux valgus angle using a goniometer, foot pain using the visual analogue scale (VAS), and walking ability evaluated by walking ability scale (WAS). These parameters were re-evaluated after 8 weeks of therapy.

The exercise program consisted of passive abduction of the hallux with traction of the first metatarsophalangeal joint and active abduction of the hallux. The subjects were asked to do the exercises 2 times daily with 10 repetitions for a period of 8 weeks. The study group was also treated with non-elastic white tape. There was a significant decrease in 
the hallux valgus angle, resting pain and walking pain in both groups. Furthermore, improvement of walking ability in the study group was noted. The treatment results of the two groups were significantly different in favour of the study group with decreases in all of the parameters. This led the authors to conclude that complex therapy combining exercise and taping produces better results in patients with hallux valgus deformity than just exercise.

Good results through taping therapy were obtained by Jeon et al. ${ }^{16)}$ in their intervention study. They assessed the pain and hallux valgus angle of fifteen patients (twenty four feet) with hallux valgus. The treatment included 15 treatments in a 4 -week period.

In a randomised clinical trial, Bek and Kurklu ${ }^{17)}$ investigated the comparative efficacy of three different conservative treatments of flexible hallux valgus: toe separator, night splints, and mobilization exercises. Forty-five participants were randomly allocated according to treatment approach into three groups of fifteen subjects. Pain and clinical manifestations of hallux valgus were assessed using the Visual Analogue Scale and Hallux Valgus Index before treatment and after the 3-month intervention. The results show that the toe separator reduced neither pain nor deformity, the night splint did not correct the deformity but relieved pain, and the mobilisation techniques helped to reduce pain and also effectively decreased flexible deformity to some extent.

Interesting results were reported by Kim et al. ${ }^{18)}$, who compared muscle activities of the abductor halluces $(\mathrm{AbdH})$ and adductor halluces (AddH) between the short foot (SF) and toe-spread-out exercises (TSO) in eighteen subjects with mild hallux valgus. They also measured the angle of their first metatarsophalangeal (MTP) joint in the horizontal plane during the SF and TSO exercises. The results suggest that the TSO exercise resulted in more beneficial effects on muscle imbalance in hallux valgus deformity, since there was a significantly greater activation of the $\mathrm{AbdH}$ in the TSO exercise than did the SF exercise, no significant difference between the SF and TSO exercises in activation of the AddH, and the angle of the first MTP joint in the horizontal plane during the TSO was significantly greater than that in the SF exercise.

Another study ${ }^{19)}$ showed the effectiveness of TSO exercise for patients with hallux valgus reporting reductions in the hallux valgus angle as well as the hallux valgus angle during active abduction, and increases in the cross-sectional area of the abductor hallucis muscle. In addition, this exercise was recommended for strengthening the medial longitudinal by $\mathrm{Heo}$ et $\mathrm{al}^{20)}$.

\section{DISCUSSION}

The studies reviewed employed various interventions and different protocols making their comparison difficult. Nonetheless all of the studies reported beneficial effects after the implementation of various physiotherapy methods. The most common benefits were reduction in pain and correction of deformity.

The outcome measures were not always well chosen. The visual analogue scale is often not an appropriate method of pain assessment. Appropriate instruments are standardized questionnaires which assess pain during certain actions or in certain positions. Also, the studies reviewed here were characterized by small numbers of participants. The largest study group consisted of 30 patients $^{8)}$. None of the studies assessed the long-term results of treatment. All of the studies examined the patients just after the end of therapy and none performed a follow up.

This literature review shows that patients with hallux valgus can be treated with physiotherapy. Physiotherapy may be used alone or in combination with other conservative treatments as a basic treatment for mild hallux valgus or as a supplement to surgical treatment in the post-operative period. In agreement with other researchers ${ }^{21)}$, it is our opinion that the physiotherapy approaches should primarily concentrate on pain relief and improvement of foot function and secondarily on the correction of deformity. Even if physiotherapy only inhibits further deformation, it should be recognized as a therapeutic success, and for patients receiving surgery, physiotherapy can prevent the occurrence of complications.

The results of conservative treatment in hallux valgus are comparable to surgical outcomes ${ }^{6,22,23)}$. Furthermore, non-operative methods do not cause many complication. An additional benefit of conservative approaches are the costs associated with surgery are avoided. Manual therapy, exercises, night splints or tapes are much cheaper and more readily available.

It was not the purpose of this study to adjudicate over which treatment, conservative or surgical, is better in cases of hallux valgus. In the author's opinion, each case should be individually analysed and the treatment should depend on the severity of the deformity and the patient's clinical symptoms. Besides, surgical interventions should be performed as a last resort, after the conservative approaches have failed to yield the desired result and symptoms such as pain persist ${ }^{21)}$.

The performance of physiotherapy for patients with hallux valgus has beneficial effects both in post-operative period and in situations when it is provided as a basic treatment. Physiotherapy, even when it does not result in correction of deformation, can still reduce pain, improve gait quality and comfort in wearing shoes. Therefore, patients with hallux valgus should not be exclusively referred for surgery but should also be considered for of the physiotherapy.

\section{REFERENCES}

1) Ferrari J: Hallux Valgus Deformity (bunion). UpToDate. 2014. (Accessed Nov 28, 2014)

2) Nix S, Smith M, Vicenzino B: Prevalence of hallux valgus in the general population: a systematic review and meta-analysis. J Foot Ankle Res, 2010, 3: 21. [Medline] [CrossRef]

3) Perera AM, Mason L, Stephens MM: The pathogenesis of hallux valgus. J Bone Joint Surg Am, 2011, 93: 1650-1661. [Medline] [CrossRef]

4) Tang SF, Chen CP, Pan JL, et al.: The effects of a new foot-toe orthosis in treating painful hallux valgus. Arch Phys Med Rehabil, 2002, 83: 17921795. [Medline] [CrossRef]

5) Coetzee JC: Scarf osteotomy for hallux valgus repair: the dark side. Foot Ankle Int, 2003, 24: 29-33. [Medline]

6) du Plessis M, Zipfel B, Brantingham JW, et al.: Manual and manipulative therapy compared to night splint for symptomatic hallux abducto valgus: an exploratory randomised clinical trial. Foot, 2011, 21: 71-78. [Medline] [CrossRef]

7) Lee KT, Park YU, Jegal H, et al.: Deceptions in hallux valgus: what to look for to limit failures. Foot Ankle Clin, 2014, 19: 361-370. [Medline] [CrossRef]

8) Schuh R, Hofstaetter SG, Adams SB Jr, et al.: Rehabilitation after hallux 
valgus surgery: importance of physical therapy to restore weight bearing of the first ray during the stance phase. Phys Ther, 2009, 89: 934-945. [Medline] [CrossRef]

9) Bryant AR, Tinley P, Cole JH: Plantar pressure and radiographic changes to the forefoot after the Austin bunionectomy. J Am Podiatr Med Assoc, 2005, 95: 357-365. [Medline] [CrossRef]

10) Kristen KH, Berger C, Stelzig S, et al.: The SCARF osteotomy for the correction of hallux valgus deformities. Foot Ankle Int, 2002, 23: 221-229. [Medline]

11) Dhanendran M, Pollard JP, Hutton WC: Mechanics of the hallux valgus foot and the effect of Keller's operation. Acta Orthop Scand, 1980, 51: 1007-1012. [Medline] [CrossRef]

12) Dhukaram V, Hullin MG, Senthil Kumar C: The Mitchell and Scarf osteotomies for hallux valgus correction: a retrospective, comparative analysi using plantar pressures. J Foot Ankle Surg, 2006, 45: 400-409. [Medline] [CrossRef]

13) Kernozek T, Roehrs T, McGarvey S: Analysis of plantar loading parameters pre and post surgical intervention for hallux vargus. Clin Biomech (Bristol, Avon), 1997, 12: S18-S19. [Medline] [CrossRef]

14) Jedynak T: Treating hallux abducto valgus conservatively through foot mobilisation techniques and exercise therapy. A case study. Podiatry Now, 2009: 12-15.

15) Bayar B, Erel S, Simsek IE: The effects of taping and foot exercises on patients with hallux valgus: a preliminary study. Turk J Med Sci, 2011, 41: 403-409.

16) Jeon MY, Jeong HC, Jeong MS, et al.: [Effects of taping therapy on the de- formed angle of the foot and pain in hallux valgus patients]. Taehan Kanho Hakhoe Chi, 2004, 34: 685-692. [Medline]

17) Bek N, Kurklu B: Comparison of different conservative treatment approaches in patients with hallux valgus. Artroplasti Artroskopik Cerrahi, 2002, 13: 90-93 (in Turkish).

18) Kim MH, Kwon OY, Kim SH, et al.: Comparison of muscle activities of abductor hallucis and adductor halluces between the short foot and toespread-out exercises in subjects with mild hallux valgus. J Back Musculoskeletal Rehabil, 2013, 26: 163-168.

19) Kim $\mathrm{MH}$, Yi CH, Weon JH, et al.: Effect of toe-spread-out exercise on hallux valgus angle and cross-sectional area of abductor hallucis muscle in subjects with hallux valgus. J Phys Ther Sci, 2015, 27: 1019-1022. [Medline] [CrossRef]

20) Heo HJ, Koo YM, Yoo WG: Comparison of selective activation of the abductor hallucis during various exercises. J Phys Ther Sci, 2011, 23: 915918. [CrossRef]

21) Wülker N, Mittag F: The treatment of hallux valgus. Dtsch Arztebl Int, 2012, 109: 857-867, quiz 868. [Medline]

22) Torkki M, Malmivaara A, Seitsalo S, et al.: Hallux valgus: immediate operation versus 1 year of waiting with or without orthoses: a randomized controlled trial of 209 patients. Acta Orthop Scand, 2003, 74: 209-215. [Medline] [CrossRef]

23) Torkki M, Malmivaara A, Seitsalo S, et al.: Surgery vs orthosis vs watchful waiting for hallux valgus: a randomized controlled trial. JAMA, 2001, 285: 2474-2480. [Medline] [CrossRef] 